\title{
What are the predictive factors in the risk and severity of chemotherapy-induced gastrointestinal toxicity?
}
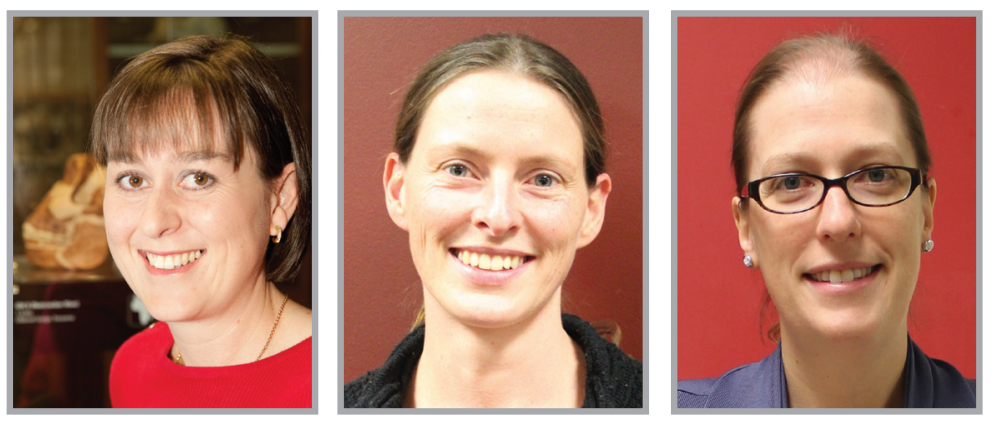

Rachel J Gibson*,1, Joanne M Bowen² \& Janet K Coller ${ }^{3}$

Damage to the mucous membranes of the GI tract by chemotherapy is known as mucositis or chemotherapy-induced gastrointestinal toxicity (CIGT). Injury can occur anywhere along the GI tract, or may be localized to a particular region, depending on the chemotherapy regimen. CIGT has huge clinical and economic implications affecting $>50 \%$ of cancer patients, and can necessitate treatment reductions and/or treatment breaks. Patients that experience severe CIGT have twice the infection risk leading to a fourfold higher chance of death and threefold longer hospital stays, compromising survival and creating a burden on patients' quality of life. Economically, US data from 2012 estimated a combined cost of US $\$ 15,500$ for each hospitalization due to severe CIGT, adding millions to healthcare expenditure [1]. Patients with CIGT typically experience severe consequences including, but not limited to, oral mucositis, increased infection rates and diarrhea [2]. Currently, there is no prophylactic intervention available for CIGT and its severity varies widely across patients receiving identical therapy. Thus there is an urgent need to be able to accurately predict which patients may develop CIGT and its severity. In light of our recent preclinical and clinical research $[3,4]$, we propose that mediators in the Toll-like receptor 4 (TLR4) signaling pathway may provide specific predictive markers of CIGT risk and severity.

\section{Key mediators of CIGT: TLR4}

\section{\& proinflammatory cytokines}

Homeostasis of the GI tract is carefully balanced by interactions between the resident microflora, epithelial barrier function and the mucosal immune system [5]. Data from our laboratories have demonstrated that CIGT disrupts the delicate balance of these three factors, leading to a substantial inflammatory response, changes in epithelial turnover and changes in microflora characteristics [6]. TLR signaling is a key component of the host-immune response, translating signals from the gut to activate innate immune responses, as well as providing links to the adaptive immune system. Our laboratory has provided compelling

\section{KEYWORDS}

- chemotherapy • gastrointestinal toxicity $\bullet$ proinflammatory cytokines - TLR 
data indicating that TLRs, in particular TLR4, modulate the pathology of CIGT [3,4]. TLR4 detects both pathogens and endogenous danger signals [7], of which, the best characterized agonist is lipopolysaccharide [8], that is present throughout the GI tract. When lipopolysaccharide binds to TLR4 in subepithelial locations, a profound inflammatory signal via the TLR pathway occurs; the end result being upregulation of proinflammatory cytokines (such as TNF, IL-1 $\beta$ and IL-6) [9].

We believe that TLR 4 activation occurs during multiple events during CIGT. Specifically we believe that there is both direct and 'off-target' activation of TLR4 by chemotherapeutic agents. In silico docking studies have shown that chemotherapies including irinotecan and $\mathrm{SN}-38$, (the major toxic metabolite of irinotecan in humans), are able to bind directly to the accessory protein of TLR4, MD2, with high specific energy [Hutchinson MR, Pers. Сомm.]. We also believe that indirect activation of TLR 4 will occur as a result of increased exposure to bacterial influx due to compromised gut barrier integrity. In support of this, our research has shown significant epithelial damage during CIGT occurrence in response

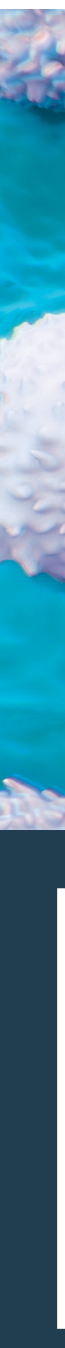

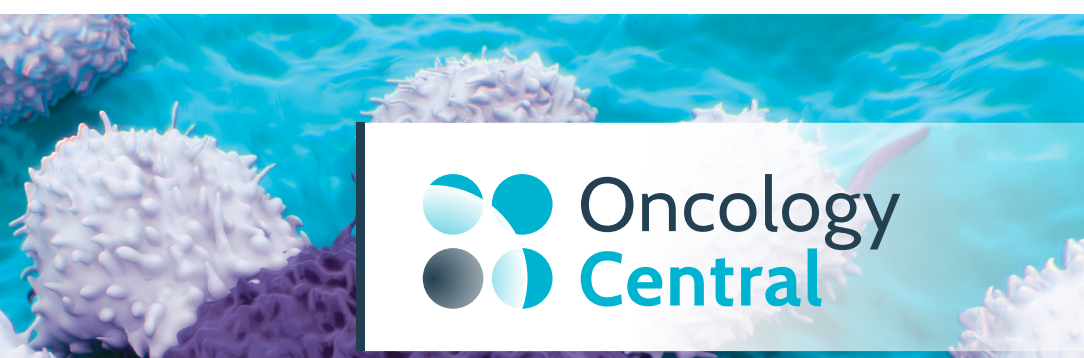

. . .
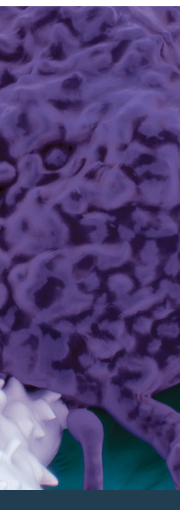

\section{ADVANCING ONCOLOGY TOGETHER}

Find out how; join our online community today to increased apoptosis and subsequent rebound hyperplasia resulting in changed epithelial barrier integrity [10]. Importantly, the time course of epithelial damage correlates with that of CIGT development observed clinically [11], highlighting the possible connection between the two events. Finally we believe that TLR4 activation will also occur from increased inflammatory mediators in a positive feedback fashion. We know that over the time course of CIGT, there is increased production of proinflammatory cytokines, in particular TNF, IL-1 $\beta$ and IL-6, and NF- $\kappa B$, a key transcriptional regulator of these proinflammatory cytokines in the gastrointestinal mucosa [12]. These are excellent markers of, and play an important role in, the pathogenesis of the chemotherapy-induced inflammatory response and CIGT.

\section{TLR4 pathway genetic variability impacts} on CIGT \& may be an excellent predictive marker

We know that the risk of cancer patients' developing CIGT depends on several variables, some of which are related to drug treatment. For example, CIGT incidence and severity is altered depending on whether the patient receives chemotherapy alone, or receives chemotherapy in combination with radiotherapy and/or the newer targeted therapies [13,14]. Other variables are related to each patient individually. For example, each individual's oral hygiene, cancer diagnosis and importantly genetic factors play key roles. Much of the previous research in this area has focused on toxicity risk associated with variability in drug-metabolizing enzyme genes that determine chemotherapeutic concentrations [15-17]. However, we believe that with the emerging role of patient innate immunity in CIGT, the variability of genes encoding mediators of the TLR4/IL-1 $\beta$ pathway are critically important markers of risk. In support of our position are studies documenting associations between proinflammatory cytokine genotypes and CIGT and other types of chemotherapy toxicity. For example, a promoter variant of the $I L-1 B$ gene, which is known to increase IL-1 $\beta$ expression was more highly expressed in patients with thrombocytopenia after treatment with chemotherapy [18]. In the same group of patients, a promoter variant of TNFA, which alters TNF expression was associated with CIGT [18]. Similar associations between TNFA and TNFB variants, again which alter TNF- $\alpha$ expression, and subsequent toxicity severity have been reported in 
patients receiving high-dose chemotherapy prior to stem-cell transplantation [19].

To prove our hypothesis that the risk of developing CIGT depends on TLR pathway genetic variability our laboratory conducted a small retrospective patient study [4]. Using TLR pathway genetic variability we successfully predicted severe CIGT risk in patients treated with 5-fluorouracilbased therapies [4]. Specifically, 34 patients (ten with severe toxicity) were genotyped for 21 loci in TLR pathway mediators and a general linear model (multivariate logistic regression) was generated incorporating genetic and demographic covariates. The final model combining genetic variability in TLR (TLR2, dimerizes with TLR4 and uses the same downstream pathway) and TNF together with cancer type (gastric and colorectal) could predict up to $87 \%$ of severe CIGT risk. Importantly, this is the first clinical study to directly link TLR genetic variability with severe CIGT and hence demonstrate the likelihood that preclinical observations linking TLR with CIGT are similar in the clinical setting.

\section{Blockade of TLR4 signaling to improve clinical management of CIGT}

In order to confirm TLR4 signaling is the key predictor of risk and severity for CIGT we need to establish that blocking the pathway will lead to reduced incidence of toxicity. In a small study using TLR4 knockout mice, our laboratory demonstrated that TLR4 plays a key role in the development of CIGT. Following irinotecan, a chemotherapy agent known to cause significant
CIGT, TLR4 knockout mice had reduced clinical symptoms compared with wild-type counterparts. Specifically these mice had significant reductions in diarrhea $(\mathrm{p}<0.0001)$ and weight loss ( $\mathrm{p}<0.021)$ and had no increase in mucosal tissue injury scores compared with wild-type mice who had significantly increased mucosa tissue injury $(p<0.002)$ [3]. These observations align with a TLR2 knockout study that demonstrated protection against doxorubicin-induced CIGT [20]. The next step to prove this theory is to block TLR2/4 signaling with an appropriate pharmacological agent that is safe to use in cancer patients.

\section{Conclusion}

We have provided strong preclinical and the first clinical evidence to suggest that TLR4 is perfectly positioned to be an accurate predictive marker of CIGT risk and severity. This strengthens the push to personalize medicine for all cancer patients.

\section{Financial \& competing interests disclosure}

This work was supported by research funding received from the Ray and Shirl Norman Cancer Research Trust and the Australian Dental Research Foundation. The authors have no other relevant affiliations or financial involvement with any organization or entity with a financial interest in or financial conflict with the subject matter or materials discussed in the manuscript apart from those disclosed.

No writing assistance was utilized in the production of this manuscript.

\section{"We have provided strong preclinical and the first \\ clinical evidence to suggest that TLR4 is perfectly positioned to be an accurate predictive marker of chemotherapy-induced gastrointestinal toxicity risk and severity. This strengthens the push to personalize medicine for all cancer patients."}

\section{References}

1 Carlotto A, Hogsett VL, Mairorini EM, Razulis JG, Sonis ST. The economic burden of toxicities associated with cancer treatment: review of the literature and analysis of nausea and vomiting, diarrhoea, oral mucositis and fatigue. Pharmacoeconomics 31(9), 753-766 (2013).

2 Gibson RJ, Keefe DM, Lalla RV et al. Systematic review of agents for the management of gastrointestinal mucositis in cancer patients. Support. Care Cancer 21, 313-326 (2013).

3 Wardill HR, Gibson RJ, Van Sebille YZA et al. TLR4 deletion attenuates irinotecan-induced gut toxicity and barrier dysfunction in the balb/c mouse: an updated report. Support. Care Cancer 23(1), S105-S106 (2015).
4 Coller JK, White IA, Logan RM et al. Predictive model for risk of severe gastrointestingal toxicity following chemotherapy using patient immune genetics and type of cancer: a pilot study. Support. Care Cancer 23(5), 1233-1236 (2015).

5 Kelly D, Campbell JI, King TP et al. Commensal anaerobic gut bacteria attenuate inflammation by regulating nuclearcytoplasmic shuttling of PPAR-gamma and RelA. Nat. Immunol. 5(1), 104-112 (2004).

6 Stringer AM, Gibson RJ, Bowen JM et al. Irinotecan-induced mucositis manifesting as diarrhoea corresponds with an amended intestinal flora and mucin profile. Int. J. Exp. Pathol. 90(5), 489-499 (2009).

7 Miyake K. Innate immune sensing of pathogens and danger signals by cell surface
Toll-like receptors. Semin. Immunol. 19(1), 3-10 (2007).

8 Guo LH, Schluesener HJ. The innate immunity of the central nervous system in chronic pain: the role of Toll-like receptors. Cell. Mol. Life Sci. 64, 1128-1136 (2007).

9 Gribar SC, Richardson WM, Sodhi CP, Hackam DJ. No longer an innocent bystander: epithelial Toll-like receptor signaling in the development of mucosal inflammation. Mol. Med. 14(9-10), 645-659 (2008).

10 Wardill HR, Bowen JM, Sultani M et al. Irinotecan disrupts tight junction proteins within the gut: implications for chemotherapy-induced gut toxicity. Cancer Biol. Ther. 15(2), 1-9 (2014).

11 Keefe DM, Brealey J, Goland GJ, Cummins AG. Chemotherapy for cancer causes 
apoptosis that precedes hypoplasia in crypts of the small intestine in humans. Gut 47(5), 632-637 (2000).

12 Logan RM, Gibson RJ, Bowen JM et al. Characterisation of mucosal changes in the alimentary tract following administration of irinotecan: implications for the pathobiology of mucositis. Cancer Chemother. Pharmacol. 62(1), 33-41 (2008).

13 Sonis ST, Elting LS, Keefe D et al. Perspectives on cancer therapy-induced mucosal injury: pathogenesis, measurement, epidemiology, and consequences for patients. Cancer 100 (9 Suppl.), 1995-2025 (2004).

14 Logan RM, Stringer AM, Bowen JM et al. Is the pathobiology of chemotherapy-induced alimentary tract mucositis influenced by the type of mucotoxic drug administered? Cancer Chemother. Pharmacol. 63(2), 239-251 (2009).

15 Deenen MJ, Cats A, Beijnen JH, Schellens JH. Part 2: pharmacogenetic variability in drug transport and phase I anticancer drug metabolism. Oncologist 16, 820-834 (2011).

16 Deenen MJ, Cats A, Beijnen JH, Schellens JH. Part 3: pharmacogenetic variability in phase II anticancer drug metabolism. Oncologist 16(6), 992-1005 (2011).

17 Cortejoso L, Lopez-Fernandez LA. Pharmacogenetic markers of toxicity for chemotherapy in colorectal cancer patients. Pharmacogenomics 13(10), 1173-1191 (2012).
18 Sakamoto K, Oka M, Yoshino S et al. Relation between cytokine promoter gene polymorphism and toxicity of 5-fluorouracil plus cisplatin chemotherapy. Oncol. Rep. 16(2), 381-387 (2006).

19 Bogunia-Kubik K, Polak M, Lange A. TNF polymorphisms are associated with toxic but not with aGVHD complications in the recipients of allogenic sibling haematopoietic stem cell transplantation. Bone Marrow Transplant. 32(6), 617-622 (2003).

20 Kaczmarek A, Brinkman BM, Heyndrickx L, Vandenabeele P, Krysko DV. Severity of doxorubicin-induced small intestine mucositis is regulated by the TLR- 2 and TLR-9 pathways. J. Pathol. 226, 598-608 (2012). 